\title{
Togitare
}

\section{UTILIZAÇÃO DA PRÁTICA BASEADA EM EVIDÊNCIAS POR ENFERMEIROS NO SERVIÇO HOSPITALAR}

\author{
Juliana de Oliveira Musse Silva' ${ }^{1}$ (1) \\ Leila Cristina Oliveira Santos ${ }^{1}$ (i) \\ Alef Nascimento Menezes ${ }^{2}$ (1) \\ Abel Lopes Neto ${ }^{1}$ (i) \\ Luciana Simões de Melo ${ }^{1}$ (i) \\ Flávia Janólio Costacurta Pinto da Silva ${ }^{3}$ (]
}

\section{RESUMO}

Objetivo: descrever as práticas, atitudes e conhecimento dos enfermeiros hospitalares sobre a Prática Baseada em Evidências.

Método: estudo descritivo, com 124 enfermeiros de um hospital universitário de Sergipe, Brasil. Foi aplicado o questionário Evidence-Based Practice Questionnaire, entre janeiro e fevereiro de 2019, e os dados analisados pelos testes de Shapiro-Wilk, Kruskal-Wallis e Post hoc.

Resultados: $86,3 \%$ dos participantes são do sexo feminino, média de idade de 34,5 anos e tempo de formação entre cinco e dez anos. A pontuação média final para a escala de Prática Baseada em Evidências e Efetividade foi de 115,9, indicando atitudes positivas dos enfermeiros relacionadas à Prática Baseada em Evidências.

Conclusão: as atitudes dos enfermeiros são embasadas em resultados científicos, entretanto, nem sempre as melhores evidências são utilizadas no subsídio das práticas profissionais. Difundir o uso adequado da pesquisa na Enfermagem contribui para a segurança do paciente, educação permanente e qualidade da assistência.

DESCRITORES: Enfermagem Baseada em Evidências; Prática Clínica Baseada em Evidências; Assistência Integral à Saúde; Enfermagem; Hospitais

\section{UTILIZACIÓN DE LA PRÁCTICA BASADA EN LA EVIDENCIA POR ENFERMEROS EN EL SERVICIO HOSPITALARIO}

\section{RESUMEN:}

Objetivo: Describir las prácticas, actitudes y conocimientos de enfermeros hospitalarios sobre Práctica Basada en la Evidencia. Método: Estudio descriptivo, con 124 enfermeros de hospital universitario de Sergipe, Brasil. Fue aplicado cuestionario Evidence-Based Practice Questionnaire, en enero y febrero de 2019. Datos analizados por tests de Shapiro-Wilk, Kruskal-Wallis y Post hoc. Resultados: $86,3 \%$ de participantes era de sexo femenino, media etaria de 34,5 años, tiempo de formación entre cinco y diez años. El puntaje promedio final para la escala de Práctica Basada en la Evidencia y Efectividad fue 115,9, indicando actitudes positivas de los enfermeros respecto de la Práctica Basada en la Evidencia. Conclusión: Las actitudes de los enfermeros se basaron en resultados científicos, aunque no siempre las mejores evidencias son utilizadas como respaldo de las prácticas profesionales. Difundir el adecuado uso de la investigación en Enfermería contribuye a la seguridad del paciente, la educación continua y la calidad de atención. DESCRIPTORES: Enfermería Basada en la Evidencia; Práctica Clínica Basada en la Evidencia; Atención Integral de Salud; Enfermería; Hospitales. 
A Prática Baseada em Evidência (PBE) refere-se à utilização dos resultados de pesquisas científicas como subsídio para a adoção de condutas durante a assistência à saúde. $O$ profissional deve utilizar evidências científicas de pesquisas com rigor metodológico, com boa validade interna e externa, para a aplicação na prática do cuidado clínico(1).

A transformação da prática tanto social quanto cultural do cuidado de enfermagem está vinculada à produção de conhecimento pela área. Desta forma, as pesquisas têm sido cada vez mais reconhecidas como essenciais aos processos de trabalho, nos campo clínico, gerencial e de ensino, por possibilitar a renovação de conhecimentos e condutas ${ }^{(2)}$.

Para uma prática baseada em evidências, o profissional de saúde deverá considerar algumas etapas. A primeira refere-se à necessidade de mudança de atitudes, e envolve a coleta de dados quanto às ações atuais desenvolvidas no serviço, identificando os problemas e elencando as possíveis intervenções e metas a serem alcançadas. A segunda etapa diz respeito à localização das melhores evidências para responder às questões levantadas. Para isso, é necessário compreender a metodologia de cada tipo de pesquisa, e planejar e implementar a busca nas fontes adequadas ${ }^{(3)}$.

$\mathrm{Na}$ terceira etapa, o profissional deverá analisar a força das evidências encontradas, avaliando criticamente a validade, benefícios e riscos para posteriormente adentrar na próxima etapa, o que inclui projetar a mudança na prática, com a identificação de recursos necessários e o planejamento dos cuidados. A quinta etapa representa a implementação e avaliação dos resultados. E, por fim, a sexta etapa busca integrar a nova prática aos padrões locais, monitorando os indicadores do processo ${ }^{(3)}$.

A PBE, na Enfermagem, constitui uma forma segura e organizada de estabelecer condutas profissionais com enfoque na identificação e solução de problemas, baseando-se nas melhores evidências científicas. Envolve as etapas de definição do problema, busca e avaliação crítica das evidências, implementação, e avaliação dos resultados. Outro aspecto a ser considerado pelo enfermeiro é a utilização das evidências de acordo com a sua competência profissional, quadro clínico e adesão do paciente, assim como a realidade dos recursos e materiais do serviço(4).

Nesse sentido, a presente pesquisa buscou descrever as práticas, atitudes e conhecimento dos enfermeiros hospitalares sobre a Prática Baseada em Evidências.

\section{MÉTODO}

Trata-se de um estudo observacional, descritivo, realizado em fevereiro de 2019, no Hospital Universitário de Sergipe-Brasil, com uma amostra de 124 enfermeiros de um total de 173. Foram incluídos todos os enfermeiros com no mínimo seis meses de atuação na instituição e que estavam lotados nas clínicas médicas, cirúrgicas, UTI (Unidade de Terapia Intensiva), pediatria, centros cirúrgicos, agência transfusional e centrais de material estéril. Foram excluídos os profissionais que se encontravam de férias, licença médica ou gestacional no período da coleta, e aqueles que deixaram de preencher alguma pergunta do questionário.

O instrumento para coleta de dados utilizado foi o Evidence-Based Practice Questionnaire (EBPQ), já validado internacionalmente e no Brasi(5), cujo objetivo é avaliar atitudes, conhecimento e implementação da PBE para assistência baseada em evidências. O instrumento possui 24 itens pontuados numa escala de um a sete (escala tipo Likert). 
escore é calculado somando-se os valores das respostas de cada uma das questões, totalizando 168 pontos, com a maior pontuação indicando atitudes mais positivas em relação à efetividade clínica e à PBE.

Os escores do questionário de Prática Baseada em Evidências e Efetividade Clínica apresentavam variação entre 1-7, sendo 1 a resposta mais negativa e 7 a mais positiva. Após a obtenção dos valores, foi realizada a divisão do questionário em três domínios: domínio 1 - "Prática Baseada em Evidências"; domínio 2 - "Atitudes relacionadas à PBE" e domínio 3 - "Conhecimentos e habilidades da PBE". Para análise, foi realizada a frequência absoluta e relativa para as variáveis categóricas qualitativas, e média e desvio padrão (DP) para as variáveis quantitativas.

A organização do banco de dados ocorreu através do programa Excel 365, categorizando variáveis qualitativas e mensurando as variáveis quantitativas. A análise estatística deu-se através do software Statistical Package for the Social Sciences, 22.0. Foi utilizado o teste Shapiro-Wilk para avaliar a normalidade das médias dos domínios 1, 2, 3 e total de cada indivíduo. Após a detecção da assimetria entre os domínios, utilizouse o teste Kruskal-Wallis e o teste Post hoc de comparação de pares para verificar se havia diferença estatística entre os grupos de graduados, especialistas e mestres, além de identificar quais grupos apresentam divergência dos valores médios.

A pesquisa foi submetida ao Comitê de Ética em Pesquisa com Seres Humanos da Universidade Federal de Sergipe e aprovada sob o parecer 2.897.833.

\section{RESULTADOS}

Do total de 124 enfermeiros, 107 são do sexo feminino (86,3\%), com média geral de $34,8( \pm 5,74)$ anos de idade, tempo de formação de cinco a dez anos $(n=75,60,5 \%)$ e com pós-graduação $(n=109,87,9 \%)$ (Tabela 1).

Tabela 1 - Caracterização dos sujeitos da pesquisa. Aracaju, SE, Brasil, 2019

\begin{tabular}{|c|c|c|}
\hline Variáveis & n (\%) & Média (DP) \\
\hline \multicolumn{3}{|l|}{ Sexo } \\
\hline Masculino & $17(13,7)$ & \multirow[t]{2}{*}{-} \\
\hline Feminino & $107(86,3)$ & \\
\hline Idade & & $34,8( \pm 5,74)$ \\
\hline \multicolumn{3}{|c|}{ Tempo de Formação } \\
\hline$<5$ anos & $3(2,4)$ & \multirow{4}{*}{-} \\
\hline $5-10$ anos & $75(60,5)$ & \\
\hline$>10$ anos & $45(36,3)$ & \\
\hline Não relatado & $1(0,8)$ & \\
\hline \multicolumn{3}{|l|}{ Grau de Formação } \\
\hline Graduação & $15(12,1)$ & \multirow{3}{*}{-} \\
\hline Especialização & $95(76,6)$ & \\
\hline Mestrado & $14(11,3)$ & \\
\hline Fonte: Autores (2020). & & \\
\hline
\end{tabular}


A pontuação média final para a escala de Prática Baseada em Evidências e Efetividade foi de 115,9 $( \pm 22,94)$. O domínio que apresentou maior média foi o Domínio $2(5,4)$, seguido do Domínio $1(4,70)$ e Domínio $3(4,60)$ (Tabela 2).

Tabela 2 - Estatística descritiva para pontuação dos domínios do questionário EBPO Aracaju, SE, Brasil, 2019

\section{Domínios}

I - Prática da Enfermagem Baseada em Evidências

II - Atitudes Relacionadas à Prática Baseada em Evidências

III - Conhecimentos e habilidades

Geral

Fonte: Autores (2020).
Média (DP) Pontuação Total

Média (DP)

No Domínio 1 do questionário (Tabela 3), os itens que obtiveram as maiores médias foram $A, B$ e D ( $\bar{x}=4.8)$, relacionados à formulação de questão norteadora para impulsionar a pesquisa, busca pelos artigos científicos e integração entre teoria encontrada e prática profissional. Já o item $C$, relacionado à frequência com que o enfermeiro analisa criticamente a literatura, obteve a menor pontuação $(4,3)$.

Tabela 3 - Estatística descritiva para os itens do Domínio 1 - Prática Baseada em Evidências. Aracaju, SE, Brasil, 2019

\section{Domínio 1}

\begin{tabular}{clc}
\hline Itens & \multicolumn{1}{c}{ Descrição } & Média (DP) \\
\hline A & $\begin{array}{l}\text { Com que frequência você formulou uma questão que pode ser claramente } \\
\text { respondida para preencher uma lacuna ou falta do seu conhecimento. }\end{array}$ & $4,8( \pm 1,60)$ \\
\hline B & $\begin{array}{l}\text { Com que frequência você buscou evidências relevantes uma vez formulada a } \\
\text { pergunta. }\end{array}$ & $4,8( \pm 1,60)$ \\
\hline C & $\begin{array}{l}\text { Com que frequência você avaliou criticamente toda a literatura encontrada com } \\
\text { base em algum critério estabelecido. }\end{array}$ & $4,3( \pm 1,70)$ \\
\hline D & $\begin{array}{l}\text { Com que frequência você integrou a evidência encontrada com o seu } \\
\text { conhecimento e experiência prévios. }\end{array}$ & $4,8( \pm 1,65)$ \\
\hline E & Com que frequência você avaliou os resultados da sua prática. & $4,7( \pm 1,72)$ \\
\hline F & Com que frequência você compartilhou esse conhecimento com colegas. & $4,7( \pm 1,71)$ \\
Fonte: Autores (2020).
\end{tabular}

No que concerne ao Domínio 2, o item I apresentou maior média de pontuação $(6,4)$, 
demonstrando uma percepção positiva por parte dos participantes quanto à aplicabilidade das evidências na rotina profissional, enquanto que o item $G$, que relaciona a carga de trabalho com o uso da PBE, obteve a menor pontuação $(4,4)$ (Tabela 4).

Tabela 4 - Estatística descritiva para os itens do Domínio 2 - Atitudes relacionadas à PBE. Aracaju, SE, Brasil, 2019

\section{Domínio 2}

\begin{tabular}{clc}
\hline Itens & \multicolumn{1}{c}{ Descrição } & Média (DP) \\
\hline $\mathrm{G}$ & $\begin{array}{l}\text { Minha carga de trabalho é muito grande para que eu me mantenha atualizado } \\
\text { com todas as novas evidências/ Novas evidências são tão importantes que eu } \\
\text { defino um tempo para isso na minha agenda de trabalho. }\end{array}$ & $4,4( \pm 1,79)$ \\
\hline $\mathrm{H}$ & $\begin{array}{l}\text { Eu me sinto desconfortável quando minha prática é questionada/ Eu acolho de } \\
\text { forma aberta os questionamentos sobre a minha prática. }\end{array}$ & $5,6( \pm 1,41)$ \\
\hline I & $\begin{array}{l}\text { Práticas baseadas em evidências são perda de tempo/ Práticas baseadas em } \\
\text { evidências são fundamentais para a prática profissional. }\end{array}$ & $6,4( \pm 1,42)$ \\
\hline J & $\begin{array}{l}\text { Eu mantenho o uso de métodos testados e confiáveis ao invés de mudar para } \\
\text { algo novo/ Minha prática tem mudado em função das evidências que tenho } \\
\text { encontrado. }\end{array}$ & $5,2( \pm 1,66)$
\end{tabular}

Fonte: Autores (2020).

Quanto ao Domínio 3, o item X, que aborda a capacidade de rever a própria prática profissional, apresentou maior média $(5,4)$, enquanto o item $K$, que se refere à habilidade de realizar a pesquisa, apresentou a menor média $(4,2)$ (Tabela 5$)$.

Tabela 5 - Estatística descritiva para os itens do Domínio 3 - Conhecimentos e habilidades. Aracaju, SE, Brasil, 2019 (continua)

\section{Domínio 3}

\begin{tabular}{clc}
\hline Itens & \multicolumn{1}{c}{ Descrição } & Média (DP) \\
\hline K & Sua habilidade em pesquisa. & $4,2( \pm 1,29)$ \\
\hline L & Sua habilidade em informática. & $4,4( \pm 1,29)$ \\
\hline M & Suas habilidades de monitoramento e revisão das práticas. & $4,3( \pm 1,21)$ \\
\hline N & $\begin{array}{l}\text { Sua capacidade de converter suas necessidades de conhecimento em uma } \\
\text { questão de pesquisa. }\end{array}$ & $4,2( \pm 1,32)$ \\
\hline O & Seu conhecimento dos principais tipos e fontes de informação existentes. & $4,4( \pm 1,23)$ \\
\hline P & Sua capacidade para identificar lacunas na prática profissional. & $4,8( \pm 1,21)$ \\
\hline Q & Seu conhecimento sobre como levantar evidências. & $4,3( \pm 1,30)$ \\
\hline R & $\begin{array}{l}\text { Sua capacidade de analisar criticamente as evidências frente aos padrões já } \\
\text { estabelecidos. }\end{array}$ & $4,5( \pm 1,28)$ \\
\hline
\end{tabular}




\begin{tabular}{cll}
\hline S & Sua capacidade de determinar quão válido é o material. & $4,6( \pm 1,36)$ \\
\hline T & Sua capacidade de determinar quão aplicável clinicamente é o material. & $4,6( \pm 1,27)$ \\
\hline U & Sua capacidade de aplicar o conhecimento a casos individuais. & $4,8( \pm 1,26)$ \\
\hline V & O compartilhamento de suas ideias e conhecimento com os colegas de trabalho. & $5,3( \pm 1,18)$ \\
\hline W & A disseminação de novas ideias sobre cuidado entre os colegas. & $5,2( \pm 1,28)$ \\
\hline X & A capacidade de rever a sua própria prática. & $5,4( \pm 1,22)$
\end{tabular}

Fonte: Autores (2020).

Analisando as questões de todos os domínios, aquele que apresentou maior pontuação foi o I. Práticas baseadas em evidências são fundamentais para a prática profissional $(6,4 \pm 1,42)$, presente no Domínio 2, ao passo que os de menores índices foram o N - Sua capacidade de converter suas necessidades de conhecimento em uma questão de pesquisa $(4,2 \pm 1,29)$ e o $\mathrm{K}$ - Sua habilidade em pesquisa $(4,2 \pm 1,32)$, ambos incluídos no Domínio 3.

Aplicando-se o teste de Kruskal-Wallis de amostras independentes, obteve-se significância estatística entre os grupos de enfermeiros graduados e mestres no que se refere aos Domínios 1 e 3, inferindo que quanto maior o grau de escolaridade, melhor o desempenho referente às práticas e habilidades necessárias para o uso das evidências. 0 mesmo não foi observado no comparativo do Domínio 2 entre os grupos, demonstrando que a atitude independe do nível de escolaridade.

\section{DISCUSSÃO}

A média final do escore do EBPQ pelos sujeitos do estudo demonstrou que os enfermeiros possuem atitudes mais positivas em relação à efetividade clínica e à prática baseada em evidências que negativas, atingindo mais de $60 \%$ da pontuação total do questionário (168). Um estudo realizado no Brasil(5) com 158 enfermeiros apresentou resultados semelhantes, com pontuação média final igual a 129,15 para o grupo composto por enfermeiros com mestrado ou doutorado e de 111,24 para o formado por enfermeiros do Hospital Público com graduação completa.

Os domínios que apresentaram as maiores médias de pontuação foram os relacionados a práticas e atitudes (1 e 2), e esses resultados corroboram com outros encontrados na literatura(5-7). Entretanto, um estudo desenvolvido com 338 enfermeiros em Portugal apresentou resultados divergentes: as subescalas que apresentaram os valores mais elevados e favoráveis à PBE foram a de "Atitudes" $(5,98 \pm 0,97)$, seguindo-se os "Conhecimentos/habilidades e competências" $(5,07 \pm 0,90)$ e as "Práticas" $(4,43 \pm 1,38)$ (8).

Vale ressaltar que, na pesquisa em questão, os itens relacionados aos conhecimentos e habilidades obtiveram os menores escores, evidenciando a dificuldade que os enfermeiros possuem para realizar a pesquisa. Isso demonstra que, embora os profissionais busquem as evidências científicas para subsidiar suas ações na assistência, não há garantia de que estas sejam as mais adequadas, uma vez que a escolha das melhores evidências está atrelada à qualidade da estratégia de busca realizada. Um dos principais obstáculos para a utilização do conhecimento produzido em enfermagem é a compreensão dos resultados de pesquisas ${ }^{(9)}$. 
A PBE caracteriza-se pela utilização dos melhores e mais atuais resultados de pesquisas disponíveis, bem como por habilidades e competências do profissional que ultrapassam aquelas consideradas como "tradicionais" para o desempenho das atividades rotineiras. Exige que o profissional consiga levantar as questões norteadoras para tomada de decisão, buscando respostas científicas pertinentes e a avaliação crítica das informações colhidas. Portanto, é necessário, por parte do investigador, rigorosidade metodológica na estratégia de busca pelas pesquisas, bem como a avaliação do desenho do estudo, sua validade interna e externa ${ }^{(8,10)}$.

Um dos resultados dessa pesquisa demonstrou maior desempenho na utilização da PBE entre enfermeiros com maior nível de especialização. Outros estudos realizados em ambientes hospitalares encontraram resultados semelhantes ${ }^{(11-15)}$.

Vale ressaltar duas importantes vertentes que influenciam a adesão da utilização efetiva da PBE no processo de trabalho pelo enfermeiro. A primeira corresponde aos fatores internos ou inerentes ao próprio profissional, que dizem respeito à importância e necessidade de adotar a pesquisa como ferramenta de atualização para a assistência, bem como às competências e habilidades necessárias para a classificação, busca e escolha das melhores evidências e uso de tecnologias de informação e comunicação em saúde. Já os aspectos externos estão relacionados ao reconhecimento e interesse do ambiente organizacional em motivar o uso da PBE na rotina dos serviços, promovendo, por exemplo, a implementação de mecanismos de capacitação(15).

Entretanto, há fatores que dificultam a implementação da PBE pela enfermagem, tais como a quantidade insuficiente de pesquisas na área, principalmente de estudos com evidência nível 1, e a falta de incentivo e cultura de pesquisa nos ambientes hospitalares. Há de se considerar que parte dos nosocômios brasileiros tem como rotina culturalmente instituída uma rígida estrutura organizacional e uma centralização de poder, que resulta em competição, individualismo e dificuldade da realização do trabalho em equipe ${ }^{(9)}$.

Para a incorporação da PBE na prática assistencial do Enfermeiro, é necessário considerar o ambiente, as características dos profissionais e as diversas estratégias que possibilitarão a inovação. Nesse sentido, sugere-se obedecer aos seguintes critérios: persuasão dos profissionais para a criatividade e originalidade, participação e apoio dos diferentes níveis organizacionais, planejamento das ações, avaliação dos resultados e decisão sobre a sua continuidade ${ }^{(1,7-8)}$.

Este estudo teve algumas limitações: surgiram algumas dúvidas quanto à interpretação de perguntas do questionário pelos participantes, sendo necessário que os pesquisadores, no momento da coleta, colaborassem para esclarecimento. No entanto, esforços foram feitos para controlar possível direcionamento das respostas, com o preenchimento do questionário validado sem interferências. Além disso, os resultados foram obtidos através da aplicação de um instrumento com perguntas fechadas e a implementação real das atitudes não pôde ser observada na prática.

Embora os enfermeiros considerem a PBE fundamental para subsidiar a assistência de enfermagem e as suas atitudes sejam embasadas em resultados de pesquisa científica no seu campo de atuação, eles demonstram dificuldades em cumprir as etapas necessárias para investigação das melhores evidências.

A aplicabilidade da pesquisa no campo de atuação prática dos enfermeiros ainda é subutilizada. Para a incorporação da PBE na prática assistencial do profissional, é necessário considerar 0 ambiente e o processo de trabalho. A PBE na enfermagem hospitalar 
proporciona maior confiabilidade das intervenções dos profissionais, consequentemente promovendo a melhoria na qualidade do cuidado. Nesse sentido, o desenvolvimento de ações que fortaleçam a formação dos enfermeiros têm sido um dos maiores desafios enfrentados nos serviços de saúde.

Dentre as estratégias para provocar a difusão dessa ferramenta científica, destacamse as oficinas teórico-metodológicas para lideranças de enfermagem e uma educação permanente dirigida por mentores, que consigam incentivar e passar segurança aos participantes. Além disso, a inclusão do tema na grade curricular dos cursos de graduação e pós-graduação em Enfermagem também constitui uma estratégia facilitadora para a adoção da PBE, à medida que transpõe a barreira entre o pesquisar e o cuidar.

\section{REFERÊNCIAS}

1. Danski MTR, Pedrolo E, Lind J, Johann DA, Rodrigues GI. Importância da prática baseada em evidências nos processos de trabalho do enfermeiro. Cienc Cuid Saude. [Internet]. 2017 [acesso em 05 jul 2018]; 16(2). Disponível em: https://www.researchgate.net/publication/320560751 Importancia da pratica baseada em evidencias nos processos de trabalho do enfermeiro Importance of evidencebased_practice in nurse's work_processes.

2. Camargo FC, Iwamoto HH, Galvão CM, Monteiro DAT, Goulart MB, Garcia LAA. Modelos para a implementação da prática baseada em evidências na enfermagem hospitalar: revisão narrativa. Texto contexto-enferm. [Internet]. 2017 [acesso em 06 jul 2018]; 26(4). Disponível em: http://dx.doi. org/10.1590/0104-07072017002070017.

3. Bosi PL. Saúde baseada em evidências. [Internet]. EAD; 2020. [acesso em 17 out 2017]. Disponível em: https://docplayer.com.br/5874120-Saude-baseada-em-evidencias-paula-lima-bosi.html.

4. Pedrosa KKA, Oliveira ICM, Feijão AR, Machado RC. Enfermagem baseada em evidência: caracterização dos estudos no Brasil. Cogitare enferm. [Internet]. 2015 [acesso 04 jul em 2018]; 20(4). Disponível em: http://dx.doi.org/10.5380/ce.v20i4.40768.

5. Rospendowiski K, Alexandre NMC, Cornélio ME. Adaptação cultural para o Brasil e desempenho psicométrico do "Evidence Based Practice Questionnaire". Acta Paul Enferm. [Internet]. 2014 [acesso em 04 jul 2018]; 27(5). Disponível em: http://dx.doi.org/10.1590/1982-0194201400068.

6. Brown CE, Wickline MA, Ecoff L, Glaser D. Nursing practice, knowledge, attitudes and perceived barriers to evidence-based practice at an academic medical center. J Adv Nurs. [Internet] 2009 [acesso em 04 jul 2018]; 65(2). Disponível em: https://doi.org/10.1111/j.1365-2648.2008.04878.x.

7. González-Torrente S, Pericas-Beltrán J, Bennasar-Veny M, Adrover-Barceló R, Morales-Asencio JM, Pedro-Goméz JD. Perception of evidence-based practice and the professional environment of Primary Health Care nurses in the Spanish context: a cross-sectional study. BMC Health Services Research. [Internet]. 2012 [acesso em 16 jul 2018]; 12(227). Disponível em: https://bmchealthservres.biomedcentral. com/track/pdf/10.1186/1472-6963-12-227.

8. Pereira RPG, Guerra ACP, Cardoso MJ da SP de O, dos Santos ATVMF, Figueiredo M do CAB de, Carneiro ACV. Validation of the Portuguese version of the evidence-based practice questionnaire. Rev. Latino-Am. Enfermagem. [Internet]. 2015 [acesso em 16 jul 2018]; 23(2). Disponível em: http://dx.doi. org/10.1590/0104-1169.0367.2561.

9. Camargo FC, Iwamoto HH, Galvão CM, Pereira G de A, Andrade RB, Masso GC. Competences and Barriers for the Evidence-Based Practice in Nursing: An Integrative Review. Rev bras enferm. [Internet]. 2018 [acesso em 16 jul 2018]; 71(4). Disponível em: http://dx.doi.org/10.1590/0034-7167-2016-0617.

10. Oliveira RM, Leitão IMT de A, Silva LMS da, Figueiredo SV, Sampaio RL, Gondim MM. Estratégias para 
promover segurança do paciente: da identificação dos riscos às práticas baseadas em evidências. Esc. Anna Nery. [Internet]. 2014 [acesso em 06 jul 2018]; 18(1). Disponível em: http://dx.doi.org/10.5935/14148145.20140018.

11. Dalhein A, Harthug S, Nilsen RM, Nortvedt MW. Factors influencing the development of evidencebased practice among nurses: a self-report survey. BMC Health Services Research. [Internet]. 2012 [acesso em 06 jul 2018]; 12(357). Disponível em: https://doi.org/10.1186/1472-6963-12-367.

12. Koehn ML, Lehman K. Nurse's perceptions of evidence-based nursing practice. J Adv Nurs. [Internet]. 2008 [acesso em 06 jul 2018]; 62(2). Disponível em: https://doi.org/10.1111/j.1365-2648.2007.04589.x.

13. Prior P, Wilkinson J, Neville S. Practice nurse use of evidence in clinical practice: a descriptive survey. Nurs Prax N Z. [Internet]. 2010 [acesso em 06 jul 2018] 26(2). Disponível em: https://www.ncbi.nlm.nih. gov/pubmed/21032971.

14. Brown CE, Ecoff L, Kim SC, Wickline MA, Rose B, Klimpel K, et al. Multi-institutional study of barriers to research utilization and evidence-based practice among hospital nurses. J Clin Nurs. [Internet]. 2010 [acesso em 10 jul 2018]; 19(13-14). Disponível em: https://doi.org/10.1111/j.1365-2702.2009.03184.x.

15. Jensen R, Guedes $E$ de $S$, Leite MMJ. Competências em informática essenciais à tomada de decisão no gerenciamento em enfermagem. Rev Esc Enferm USP. [Internet]. 2016 [acesso em 10 jul 2018]; 50(1).

Disponível em: https://doi.org/10.1590/S0080-623420160000100015.

COMO REFERENCIAR ESTE ARTIGO:

Silva J de OM, Santos LCO, Menezes NA, Lopes Neto A, Melo LS de, Silva FJCP da. Utilização da prática baseada em evidências pelo enfermeiro no serviço hospitalar. Cogitare enferm. [Internet]. 2021 [acesso em "colocar data de acesso, dia, mês abreviado e ano"]; 26. Disponível em: http://dx.doi.org/10.5380/ce.v26i0.67898.

Recebido em: 08/07/2019

Aprovado em: 15/09/2020

Autor Correspondente:

Juliana de Oliveira Musse Silva

Universidade Federal de Sergipe - Aracaju, SE, Brasil

E-mail: julimusse@hotmail.com

Contribuição dos autores:

Contribuições substanciais para a concepção ou desenho do estudo; ou a aquisição, análise ou interpretação de dados do estudo - JOMS, LCOS, ANM, ALN, LSM

Elaboração e revisão crítica do conteúdo intelectual do estudo - LCOS, ALN, LSM, FJCPS

Aprovação da versão final do estudo a ser publicado - JOMS, FJCPS

Responsável por todos os aspectos do estudo, assegurando as questões de precisão ou integridade de qualquer parte do estudo - JOMS

Copyright () 2021 Este é um artigo em acesso aberto distribuído nos termos da Licença Creative Commons Atribuição, que permite o uso irrestrito, a distribuição e reprodução em qualquer meio desde que o artigo original seja devidamente citado. 